$10-27-2016$

\title{
Response of a Doublet to a Nearby DC Electrode of Uniform Potential
}

Christopher L. Wirth

Cleveland State University

Sri Harsha Nuthalapati

Follow this and additional works at: https://engagedscholarship.csuohio.edu/encbe_facpub

Part of the Chemical Engineering Commons

How does access to this work benefit you? Let us know!

\section{Original Citation}

Wirth CL, Nuthalapati SH. Response of a doublet to a nearby dc electrode of uniform potential. Phys Rev E. 2016;94:042614.

\section{Repository Citation}

Wirth, Christopher L. and Nuthalapati, Sri Harsha, "Response of a Doublet to a Nearby DC Electrode of Uniform Potential" (2016). Chemical \& Biomedical Engineering Faculty Publications. 124.

https://engagedscholarship.csuohio.edu/encbe_facpub/124

This Article is brought to you for free and open access by the Chemical \& Biomedical Engineering Department at EngagedScholarship@CSU. It has been accepted for inclusion in Chemical \& Biomedical Engineering Faculty Publications by an authorized administrator of EngagedScholarship@CSU. For more information, please contact library.es@csuohio.edu. 


\title{
Response of a doublet to a nearby dc electrode of uniform potential
}

\author{
Christopher L. Wirth* and Sri Harsha Nuthalapati \\ Chemical and Biomedical Engineering Department, Washkewicz College of Engineering, Cleveland State University, \\ 2121 Euclid Avenue, Cleveland, Ohio 44115, USA
}

(Received 17 June 2016; revised manuscript received 28 September 2016; published 27 October 2016)

\begin{abstract}
The electric-field-assisted directed assembly of spherical colloidal particles near an electrode has been studied for nearly three decades. Recently, focus has shifted to the electric-field-assisted assembly and propulsion of nonspherical (i.e., anisotropic) particles. This paper describes calculations and results for a doublet of asymmetric $\zeta$ potential and size responding to a dc electric field. The doublet experienced a net vertical force that depended on both the asymmetry in $\zeta$ potential and lobe size. In addition, the doublet experienced a net lateral force perpendicular to the applied electric field. The lateral force depended on the difference in $\zeta$ potential of the two lobes, the lobe size asymmetry, and also the angle of inclination of the doublet. The net force was used to predict an apparent lateral velocity, which was found to be perpendicular to the applied electric field. In addition, the particle experienced rotation from a net torque that depended on the lobe size asymmetry and also the angle of inclination of the doublet. The magnitude of the predicted velocity was of the same order of magnitude as has been observed for particles responding to ac electric fields in experiments. These results demonstrate that lobe $\zeta$ potential, lobe size, and orientation of a colloidal doublet can be tuned to achieve propulsion.
\end{abstract}

DOI: 10.1103/PhysRevE.94.042614

\section{INTRODUCTION}

The directed assembly of micrometer-scale colloidal particles with an external electric field has potential application in a range of industries, from advanced materials to sensing and sorting in chemical and biological environments [1-6]. Consequently, work over the past three decades in this area has sought to understand both the fundamental aspects and technological obstacles in the response of colloidal particles to external dc and ac electric fields [7-31]. There has been a focused effort to understand the response of spherical colloidal particles that are near, but not adhered to, a planar electrode. The typical experiment consists of a suspension of micrometer-scale polystyrene spheres dispersed in fluid between parallel plate electrodes. The polystyrene particles, which have a specific gravity of $\sim 1.055$, settle near the bottom plate of the parallel plate arrangement. An electric field is generated by polarizing the bottom electrode with respect to the top electrode, inducing an electric field that is nominally perpendicular to the electrodes. Ensembles of particles will tend to form ordered close-packed arrays (hexagonal closepacked or random close-packed, for instance) or other ordered arrays with a center-to-center distance that is many particle diameters. These structures arise from either a net-attractive or net-repulsive interparticle force that particles feel in response to the electric field.

The origin of the force depends on a variety of experimental conditions, including electric field strength and frequency [31,32], particle surface chemistry [26], and even electrolyte type and concentration [7,26]. At dc conditions, the electric field acts on the mobile charge in the diffuse layer of the particle's electric double layer to produce equilibrium charge electro-osmotic (ECEO) flow [13]. The toroidal flow field entrains neighboring particles to cause aggregation (repulsion) in the case of a particle with a negative surface

*Corresponding author: c.wirth@csuohio.edu charge and positive (negative) electric field [9,12]. Particle assembly in ac electric fields is a more nuanced problem that still has unanswered questions. Although an ac electric field causes electro-osmotic flow along the particle surface at ac conditions, there will also be flow along the electrode surface as a consequence of both induced and equilibrium charge electro-osmosis. The combination of these flow fields will induce particles to experience changes in elevation (in the $z$ axis). Work from multiple groups [8,15,16,19,21-26] has shown the profound importance of this change in particle height in the $z$ axis on the assembly process in the $x y$ plane. Recent work has even shown that an ensemble of particles will achieve a bimodal distribution of heights in response to an electric field [8]. Moreover, both groups have shown the apparent importance of electrolyte type and concentration over the past decade $[7,26]$.

Until recently, work in this area has focused on the response of geometrically isotropic particles (i.e., spheres) to an applied electric field. Innovative new techniques for the fabrication of geometrically (i.e., nonspherical) or chemically (i.e., nonuniform) anisotropic particles now provide new particles that have an extra degree of control for the synthesis of colloidal assemblies [33-37]. The additional control arises from the relationship between geometry and pairwise interaction force for anisotropic colloidal particles in an applied electric field. A geometrically anisotropic particle exposed to an external electric field will engender anisotropic pairwise interparticle interactions. Colloidal assemblies (either crystalline or noncrystalline) fabricated from anisotropic particles with spatially dependent interactions will have more intricate and complex microstructure than those fabricated from ensembles of isotropic particles. For instance, small groups of doublets will assemble into chiral clusters, while large numbers of doublets form intricate microstructure [35,37].

Moreover, individual anisotropic particles have been shown to respond quite differently than isotropic particles in an electric field. When near an ac electrode, a single doublet will 
propel in the direction normal to the applied electric field [37]. Propulsion arises from an asymmetric flow field associated with each lobe of the doublet. Doublets of unequally sized lobes but equal $\zeta$ potential will propel. Likewise, doublets with unequal $\zeta$ potential but equally sized lobes will propel. Propulsion speed depends on the square of the electric field $\sim E^{2}$ and the inverse of the electric field frequency $\omega^{-1}$, indicating that the mechanism arises from an induced charge electrokinetic phenomenon. This work, on both ensembles and individual doublets, has reinforced that anisotropic particles responding to an electric field will be useful for the fabrication of advanced materials.

This paper concerns the response of a colloidal doublet to a dc electric field. The flow and electric field were numerically calculated for both homo- and heterodoublets near, but not adhered to, a powered dc electrode. Numerical calculations were conducted because of the geometric complexity of the problem. The results show that the force on the doublet depends on the relative size of lobes, the lobe $\zeta$ potential, and the lobe orientation. A heterodoublet will experience a net force in the $x y$ plane that is perpendicular to the applied electric field. The apparent velocity at typical dc electric field conditions was of the same magnitude as what has been observed in experiments for doublets responding to an ac electric field.

\section{THEORY}

\section{A. The response of an isotropic particle to a nearby powered electrode}

Consider the spherical particle shown above in Fig. 1(a). The particle has a radius $R_{1}$ and equilibrium zeta potential $\zeta_{p}$. An equilibrium $\zeta$ potential is most often the result of bound charge on the surface of a particle, for instance, a sulfate or carboxyl group, although there are other ways in which surfaces may acquire charge [38]. A powered electrode will have an electric field that is nominally perpendicular to the electrode surface. When the particle is placed nearby a powered electrode as shown in Fig. 1(a), the electric field

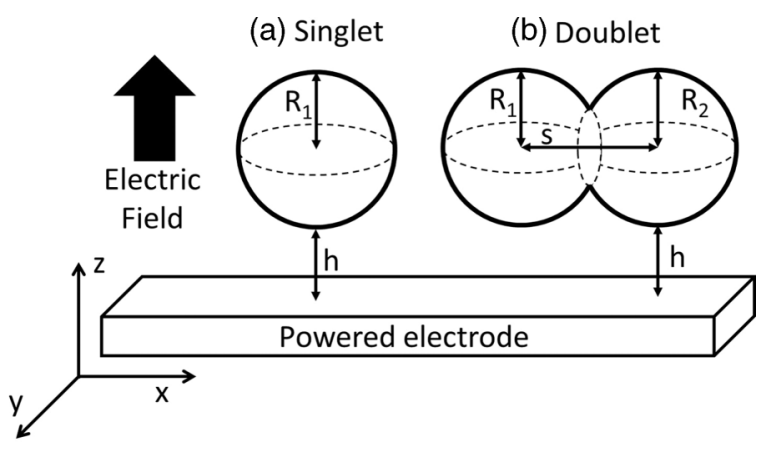

FIG. 1. (a) Singlet and (b) doublet neighboring a powered electrode. The singlet has radius and separation distance $R_{1}$ and $h$, respectively. The doublet has radii $R_{1}$ and $R_{2}$, separation distance $h$, and center-to-center distance $s$. The doublet separation distance was defined as the distance between the electrode and closest surface (for example, between the electrode and bottom of the largest lobe of a heterodoublet with $R_{1} \neq R_{2}$ ). The center-to-center distance was fixed $s /\left(R_{1}+R_{2}\right)=0.9$. acts on the mobile charge in the equilibrium electric double layer enveloping the particle. The resulting ECEO toroidal flow field has a strength and direction that depends on the sign and magnitude of the electric field and the $\zeta$ potential of the particle. When the electric field is positive and the equilibrium $\zeta$ potential of the particle is negative, flow will be driven up and along the surface of the particle. The flow field is capable of entraining neighboring particles, thereby causing particle aggregation or separation in electric fields.

In addition to inducing a toroidal flow field to entrain neighboring particles, the ECEO flow field will act to force the particle either upward or downward. When the electric field is positive and the equilibrium $\zeta$ potential of the particle is negative, there will be an effective downward force towards the boundary [39]. In the absence of the boundary (i.e., in the bulk fluid), the force on the particle will be balanced by viscous drag and the particle will become "force-free." However, when nearby the boundary, the downward electrophoretic force is balanced by strong electrostatic repulsion from the electric double layer on the electrode. The force on the particle depends sensitively on the electrode conditions; an electrode with a uniform potential will induce flow along only the particle surface, without electro-osmotic flow along the electrode surface. However, electro-osmotic flow is driven along an electrode that has a gradient in potential (i.e., not at uniform potential conditions). This additional flow along the electrode causes a difference in the height dependence of the force in the two limits. This force was used as a probe for the high throughput evaluation of electrocatalysts [3,4,39].

Rock et al. [40] calculated the force on a spherical particle at the uniform potential limit (no flow along the electrode), the uniform current limit (maximal flow on electrode), as well as the transitional regime. The electrophoretic force on the particle in the limit of uniform potential is given by $[9,40]$

$$
\begin{aligned}
F_{\mathrm{ep}} & =6 \pi \varepsilon R_{1} \zeta_{1} E_{\infty} C(h), \\
C(h) & =\frac{h+1.554 R_{1}}{h+0.300 R_{1}},
\end{aligned}
$$

where $\varepsilon$ is the dielectric permittivity of the fluid, $E_{\infty}$ is the nominal applied electric field, and $C(h)$ is the hindrance factor because of the nearby boundary at uniform potential conditions. Equation (1) applies to a particle with an infinitesimally small Debye layer as compared to the radius of the particle, or in the limit of $\kappa R_{1} \rightarrow \infty$, where $\kappa$ is the Debye parameter. The value of $C(h)$ approaches 1 when the particle moves away from the boundary, $h / R_{1} \rightarrow \infty$. This can be considered the bulk limit, such that $F_{\text {ep }}=6 \pi \varepsilon R_{1} \zeta_{p} E_{\infty}$ is the electrophoretic force on the particle far from the boundary.

\section{B. The response of a doublet to a powered electrode}

The doublet shown in Fig. 1(b) is comprised of lobes 1 and 2, with radii $R_{1}$ and $R_{2}$ and equilibrium zeta potentials $\zeta_{1,1}$ and $\zeta_{1,2}$. The center-to-center distance of the two lobes is $s$. The ratio $s /\left(R_{1}+R_{2}\right)$ was set for all calculations $=0.9$. 
A "homodoublet" is when the lobes are identical $\left(R_{1}=R_{2}\right.$ and $\left.\zeta_{1,1}=\zeta_{1,2}\right)$, while a "heterodoublet" is when either the size or $\zeta$ potential of the lobes differ $\left(R_{1} \neq R_{2}\right.$ or $\left.\zeta_{1,1} \neq \zeta_{1,2}\right)$. Either homo- or heterodoublets can be prepared via direct synthesis [34] or controlled flocculation techniques [41]. The hydrodynamic force on an overlapping doublet has been previously studied [42]. The authors provided a hydrodynamic force, and the electrophoretic force was obtained for the work presented herein by substitution of a suitable slip velocity. The electrophoretic mobility depended on the orientation of the doublet relative to the electric field, with the two limits being parallel and perpendicular to the applied electric field. The electrophoretic mobility of a doublet in the bulk was used to scale the results, providing an internal check for the simulation protocol.

\section{NUMERICAL METHOD}

\section{A. Description of numerical technique}

The fluid mechanics and electrokinetic equations were simultaneously solved for a spherical particle, homodoublet, or heterodoublet in a cube domain. The size of the particle varied between $3-\mu \mathrm{m}$ radius for a single spherical particle to 11.4- $\mu \mathrm{m}$ total width for a doublet with equal-sized lobes of $3-\mu \mathrm{m}$ radius and overlap $s /\left(R_{1}+R_{2}\right)=0.9$. The majority of simulations were conducted with a doublet that had lobes approximately $3 \mu \mathrm{m}$ in size. The simulation box for most cases was $1000 \mu \mathrm{m}$ on each side, although the box was increased in size to $2000 \mu \mathrm{m}$ on each side for the calculations of a colloidal particle moving far away from the boundary and decreased in size to $900 \mu \mathrm{m}$ for situations when higher mesh density was required.

Simulations were conducted in the thin Debye layer regime, where both the particle radius and gap between the particle and electrode are large as compared to the solution's Debye length. Note that we are referring to the equilibrium electric double layer being thin and not an induced electric double layer, as there will be negligible accumulation of induced charge during the passage of Faradaic current, as described below. There is no free charge in the fluid in this limit (i.e., the fluid is electrically neutral), and Laplace's equation can be solved with current conservation to obtain

$$
E=-\nabla \phi
$$

which relates the nominal electric field to the gradient in potential. The electrode, which was the bottom boundary in all simulations, had a uniform potential defined by the desired electric field, $\phi=E_{\infty} \times L$, where $L$ was the box side length. The electric field for all data presented herein was either $0.1 \mathrm{~V} / \mathrm{m}$ or $70 \mathrm{~V} / \mathrm{m}$. The dc electric field will be a consequence of the electrochemical couple in solution, which is electrolysis in the case of water with added salt, provided there is sufficient overpotential to drive the reaction (see Eqs. (20) and (21) in [39]). Current resulting from an electrochemical reaction is referred to as "Faradaic current," with the relationship between potential and current density (and, by extension, electric field) nonlinear at these conditions. A typical experiment at dc conditions will have an electric field strength between $50 \mathrm{~V} / \mathrm{m}$ and $100 \mathrm{~V} / \mathrm{m}$. This electric field will be achieved by applying an $\sim 1 \mathrm{~V}$ vs $\mathrm{Ag} / \mathrm{AgCl}$ reference electrode (at potentiostatic conditions) or $0.25 \mathrm{~A} / \mathrm{m}^{2}$ (at galvanostatic conditions) in an electrolyte solution with a conductivity of $30 \mu \mathrm{S} / \mathrm{cm}$ and electrode separation distance of $\sim 1 \mathrm{~mm}$. It is important to note that the results presented herein were scaled such that predictions could be applied to any electric field strength or sign. The upper surface of the box was ground $(\phi=0 \mathrm{~V})$. The surface of the particle and the remaining four walls of the box were insulating. A uniform potential boundary condition eliminates any possibility of gradients in potential in the plane of the electrode and therefore eliminates electro-osmotic flow generated along the electrode surface. Allowing for gradients in the potential along the electrode could be achieved by enforcing a uniform current density boundary condition (or a condition intermediate to the two limits). The particle itself may also induce gradients in potential along the electrode. The influence of the particle on the electric field very close to the electrode boundary was measured for a doublet at the shortest gap, $H=0.1$. The electric field in the $x$ or $y$ direction was less than $1 \%$ of the nominal electric field in the $z$ direction within $250 \mathrm{~nm}$ of the electrode in the worst case scenario.

The inertia terms of the Navier-Stokes equations were neglected because the size of the particles and associated fluid

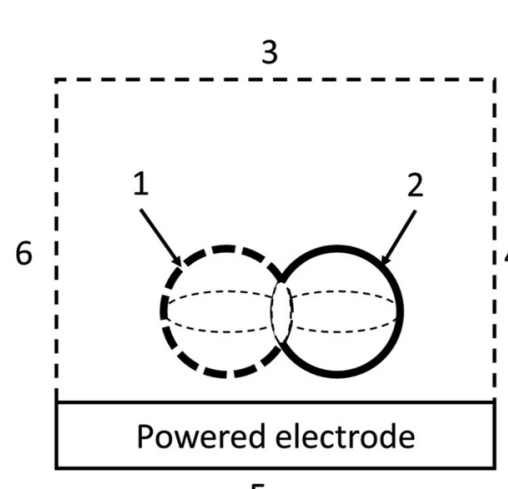

5

\begin{tabular}{|c|c|c|c|c|}
\hline Boundary & Description & \multicolumn{3}{|c|}{ Boundary Condition } \\
\hline \multirow{2}{*}{1} & \multirow{2}{*}{ Lobe 1} & Electrical: & Insulating & $\mathbf{n} \cdot \mathbf{D}=0$ \\
\hline & & Fluid: & Slip Velocity & $u_{t}=-\varepsilon \zeta_{L, 1} E_{t} / \mu$ \\
\hline \multirow{2}{*}{2} & \multirow{2}{*}{ Lobe 2} & Electrical: & Insulating & $\mathbf{n} \cdot \mathbf{D}=0$ \\
\hline & & Fluid: & Slip Velocity & $u_{t}=-\varepsilon \zeta_{1,2} E_{t} / \mu$ \\
\hline \multirow{2}{*}{3} & \multirow{2}{*}{ Top Electrode } & Electrical: & Ground & $\phi_{\mathrm{t}}=0 \mathrm{~V}$ \\
\hline & & Fluid: & Open & Normal Stress $=0$ \\
\hline \multirow{2}{*}{4} & \multirow{2}{*}{ Right Side } & Electrical: & Insulating & $\mathbf{n} \cdot \mathbf{D}=0$ \\
\hline & & Fluid: & Open & Normal Stress $=0$ \\
\hline \multirow{2}{*}{5} & \multirow{2}{*}{ Bottom Electrode } & Electrical: & Uniform Potential & $\phi_{\mathrm{b}}=\mathrm{E}_{\infty}{ }^{*} \mathrm{~L}$ \\
\hline & & Fluid: & No slip & $\mathbf{u}=0$ \\
\hline \multirow{2}{*}{6} & \multirow{2}{*}{ Left Side } & Electrical: & Insulating & $\mathbf{n} \cdot \mathbf{D}=0$ \\
\hline & & Fluid: & Open & Normal Stress $=0$ \\
\hline \multirow{2}{*}{7 (not pictured) } & \multirow{2}{*}{ Front } & Electrical: & Insulating & $\mathbf{n} \cdot \mathbf{D}=0$ \\
\hline & & Fluid: & Open & Normal Stress $=0$ \\
\hline \multirow{2}{*}{8 (not pictured) } & \multirow{2}{*}{ Back } & Electrical: & Insulating & $\mathbf{n} \cdot \mathbf{D}=0$ \\
\hline & & Fluid: & Open & Normal Stress $=0$ \\
\hline
\end{tabular}

FIG. 2. Boundary conditions for doublet simulation. The boundary conditions for the singlet simulation were identical, with the only exception being the absence of lobe 2 . 
velocities were small. The fluid flow in the three-dimensional domain was calculated with the following equations:

$$
\begin{gathered}
-\nabla p+\mu \nabla^{2} u=0, \\
\nabla \cdot u=0,
\end{gathered}
$$

which are the Stokes equation and continuity, respectively. Finally, the electrokinetic slip velocity was set on the surface of the particle,

$$
u_{t}=-\frac{\varepsilon \zeta_{p} E_{t}}{\mu},
$$

where $u_{t}$ is the tangential velocity, $\zeta_{p}$ is the zeta potential of the particle or lobe $\left(\zeta_{1}\right)$, and $E_{t}$ is the tangential electric field. The bottom boundary had a no-slip boundary condition, while the remaining five walls of the simulation box were open. All boundary conditions are summarized in Fig. 2 shown below. A finite element solver (COMSOL) was used to solve the above equations with associated domain and boundary conditions with $P 1+P 1$ discretization. The meshing was done under extremely fine mesh conditions near the particle and extra coarse mesh in the bulk.

\section{RESULTS \& DISCUSSION}

\section{A. Test case: A single isotropic particle}

The numerical solution technique was first tested by solving for the electric and flow fields on a single spherical particle and subsequently calculating the force as a function of height. Figure 3 shows the scaled forces $F_{S}$ in each direction as a function of scaled height $H$ in comparison to the analytical expression [9,40] for the force shown in Eqs. (1) and (2). The calculated force $F_{\text {calc }}$ was scaled with the force on a sphere in the bulk, and the separation distance $h$ was scaled with

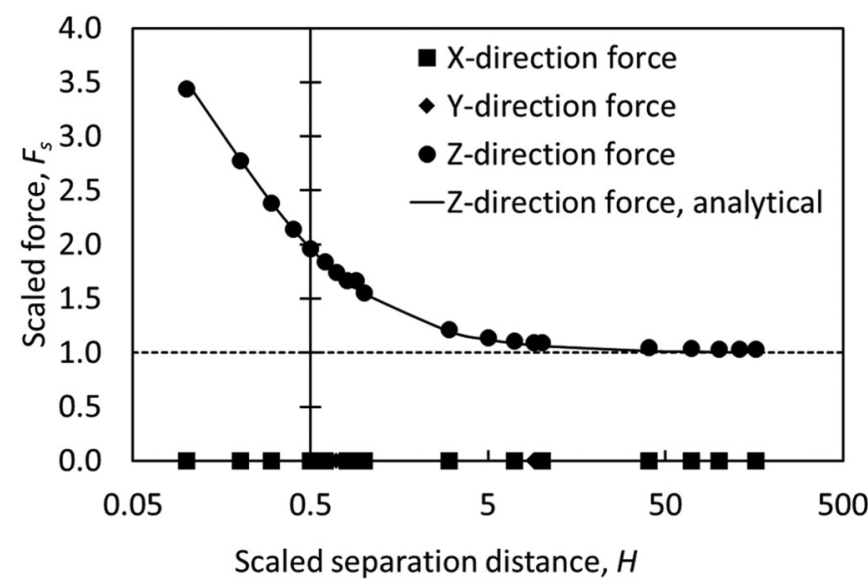

FIG. 3. Test case: Force on a singlet approaching an electrode of uniform potential. The force on single particle compared with an analytical solution $[9,40]$ that was previously published. The calculated force $F_{\text {calc }}$ was scaled with the force on a sphere in the bulk and the separation distance $h$ was scaled with the diameter of the sphere, i.e., $F_{s}=F_{\text {calc }} / 6 \pi \varepsilon R_{1} \zeta_{1} E_{\infty}$ and $H=h / 2 R_{1}$, respectively. The magnitude of the vertical force on the particle increased as separation distance decreased, and the lateral force components were zero. the diameter of the sphere, i.e., $F_{s}=F_{\text {calc }} / 6 \pi \varepsilon R_{1} \zeta_{p} E_{\infty}$ and $H=h / 2 R_{1}$, respectively.

As expected, the lateral force components in the $x$ and $y$ directions were zero, while the magnitude of the force in the $z$ direction increased as the separation distance decreased. These results were anticipated because there are no mechanisms to break the symmetry of the flow field in either the $x$ or $y$ directions (i.e., the problem is axisymmetric). A single spherical particle does not experience a lateral force when moved from the center because the electrodes extend (in the $x y$ plane) far beyond the radius of the particle. An experiment typically uses electrodes that are $\sim 1-10 \mathrm{~cm}$ wide and long, while the particle radius is typically $\sim 1-10 \mu \mathrm{m}$. This means that, even in the worst case scenario, the electrode width is 1000 larger than the particle radius, and the applied electric field (in the absence of the particle) is normal to top and bottom electrode. This physical rationale is in agreement with experiments, which have not reported deterministic lateral motion of a single spherical or symmetric doublet particle in response to an electric field. The signs of both the unscaled force and $F_{\infty}$ were negative, thereby producing a positive value for the scaled force in the $z$ direction. Note, however, that the direction of the vertical force is downward. The origin of the force is the ECEO flow field resulting from the interaction of the electric field with the positively charged diffuse cloud associated with the negatively charged particle. A positive electric field will cause flow along the particle in the $(+) z$ direction, thereby imparting a downward force to the particle. In practice, this force is balanced by a strong electrostatic repulsion that allows for the particle to remain mobile. The particle will deposit if electrostatic repulsion is insufficient to balance the downward electrophoretic force. The close agreement between the numerical results and analytical solution verifies the numerical scheme for calculating the force on a doublet.

\section{B. A doublet approaching the boundary}

The electric and flow fields on a doublet were calculated for both a homodoublet $\left(R_{1}=R_{2}\right.$ and $\left.\zeta_{1,1}=\zeta_{1,2}\right)$ and a heterodoublet $\left(R_{1} \neq R_{2}\right.$ and/or $\left.\zeta_{1,1} \neq \zeta_{1,2}\right)$ upon approach to a powered electrode as per the boundary conditions described in Sec. III A. Figure 4 shows the scaled force components on a homodoublet as a function of separation distance from the powered electrode for both perpendicular and parallel orientations. The perpendicular orientation is when the particle centers are at fixed position in the $x$ axis, while the parallel orientation is when the particle centers are at fixed position in the $z$ axis (i.e., equal center height). The forces reported in Fig. 4 were scaled with the value of the force on the doublet in the bulk.

Figure 4 shows that, as with the spherical particle, the vertical force on a homodoublet was nonzero and increased upon approach to the boundary from a value of 1 at $H \gg 1$. The lateral force components in the $x$ and $y$ directions were zero because homodoublets do not have the necessary anisotropy in shape or $\zeta$ potential to create a break in symmetry in the associated flow field. Although the value of the scaled forces for each colloid were of the same order of magnitude, the values were not equal for different orientations, with 


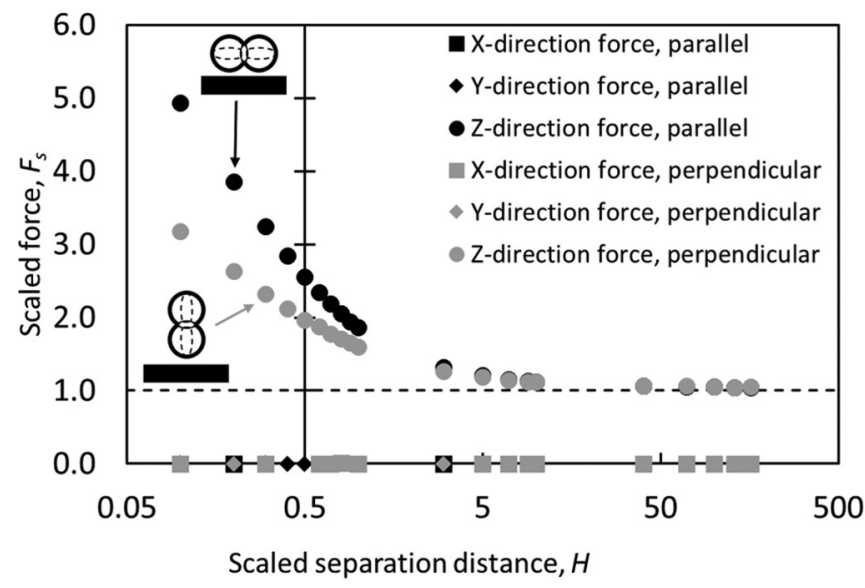

FIG. 4. Force on a homodoublet approaching an electrode of uniform potential. The force on a homodoublet scaled with the force on a homodoublet in the bulk as a function of separation distance, which was scaled with the summation of the radii of lobes. The vertical force on the homodoublet increased as separation distance decreased and the lateral force components were zero.

the parallel orientation experiencing a larger force than the perpendicular orientation. As was shown previously [25] with a sphere approaching a powered electrode, the major contribution to the net vertical force is the pressure in the gap between the particle and electrode. The length of the gap over which the pressure acts is greater for the homodoublet oriented parallel to the electrode, resulting in a greater vertical force.

\section{A doublet near a boundary}

The force on a heterodoublet $\left(R_{1}=R_{2}\right.$ and $\left.\zeta_{1,1} \neq \zeta_{1,2}\right)$ at fixed separation distance $H=0.1$ was calculated. The $\zeta$ potential of lobe 1 was systematically changed from $-100 \mathrm{mV}$ to $100 \mathrm{mV}$ and the $\zeta$ potential of lobe 2 was fixed at $-100 \mathrm{mV}$. The calculated force $F_{\text {calc }}$ was scaled with the force on a homodoublet in the bulk. Figure 5 shows the force components, with the $z$ force in Fig. 5(a) and the $x$ and $y$ forces in Fig. 5(b). The vertical force shown in Fig. 5(a) on the doublet decreased as the $\zeta$ potential of lobe 1 increased from $-100 \mathrm{mV}$ to $100 \mathrm{mV}$, while Fig. 5(b) shows the magnitude of the lateral force in the $x$ direction increased as the $\zeta$ potential of lobe 1 increased from $-100 \mathrm{mV}$ to $100 \mathrm{mV}$. These results show that both forces (in the $z$ and $x$ direction) scale linearly with the difference in $\zeta$ potential at fixed $H$. The largest vertical force was calculated for when the difference in $\zeta$ was zero $\left(\zeta_{1,1}-\zeta_{1,2}=0, \zeta_{1,1}=\zeta_{1,2}\right)$ and the largest lateral force was calculated to be when the difference in $\zeta$ potential was large $\left(\zeta_{1,1}-\zeta_{1,2}=200 \mathrm{mV}\right)$. The net force in the $z$ direction on the doublet was a consequence of electro-osmotic flow driven upward along each particle, thereby forcing the particle downward, as is the case for a single particle (see Fig. 3). The $z$ direction force was maximized at equal lobe $\zeta$ potential conditions $\left(\zeta_{1,1}-\zeta_{1,2}=0, \zeta_{1,1}=\zeta_{1,2}\right)$ because the direction of the flow neighboring each lobe was upward and along the surface of the lobe, acting in the same direction. The origin of the net force in the $x$ direction was also the electro-osmotic flow driven along the particle. However, the break in symmetry between the ECEO flow driven along lobe 1 and lobe 2 produced a net force. When the $\zeta$ potential difference was large, i.e., when $\zeta_{1,1}=100 \mathrm{mV}$ and $\zeta_{1,2}=-100 \mathrm{mV}$, electroosmotic flow driven along lobe 1 was downward along the surface of the lobe, while electro-osmotic flow driven along the surface of lobe 2 was upward. The net force in the $y$ direction was zero because the asymmetry in $\zeta$ potential was only in the $x$ direction. Note that the response of the doublet in position will be opposite that of the sign of the force-a particle will move in the negative direction for a positive force and vice versa. Thus, a heterodoublet of different $\zeta$ will tend to move in the direction of negative $\zeta$ potential for a positive dc electric field.

The force on a heterodoublet with both asymmetric lobe size and $\zeta$ potential $\left(3 R_{1}=2 R_{2}\right.$ and $\left.\zeta_{1,1} \neq \zeta_{1,2}\right)$ was calculated and the results are shown in Fig. 6. As was the case for a heterodoublet with only an asymmetric $\zeta$ potential, Fig. 6(a) shows that the vertical force on a heterodoublet with asymmetric lobe size increased as the $\zeta$ potential of lobe 1 decreased from $100 \mathrm{mV}$ to $-100 \mathrm{mV}$. The net vertical force on the heterodoublet with asymmetric lobe size linearly scaled with the $\zeta$ potential of lobe 1 , but with a slope different from
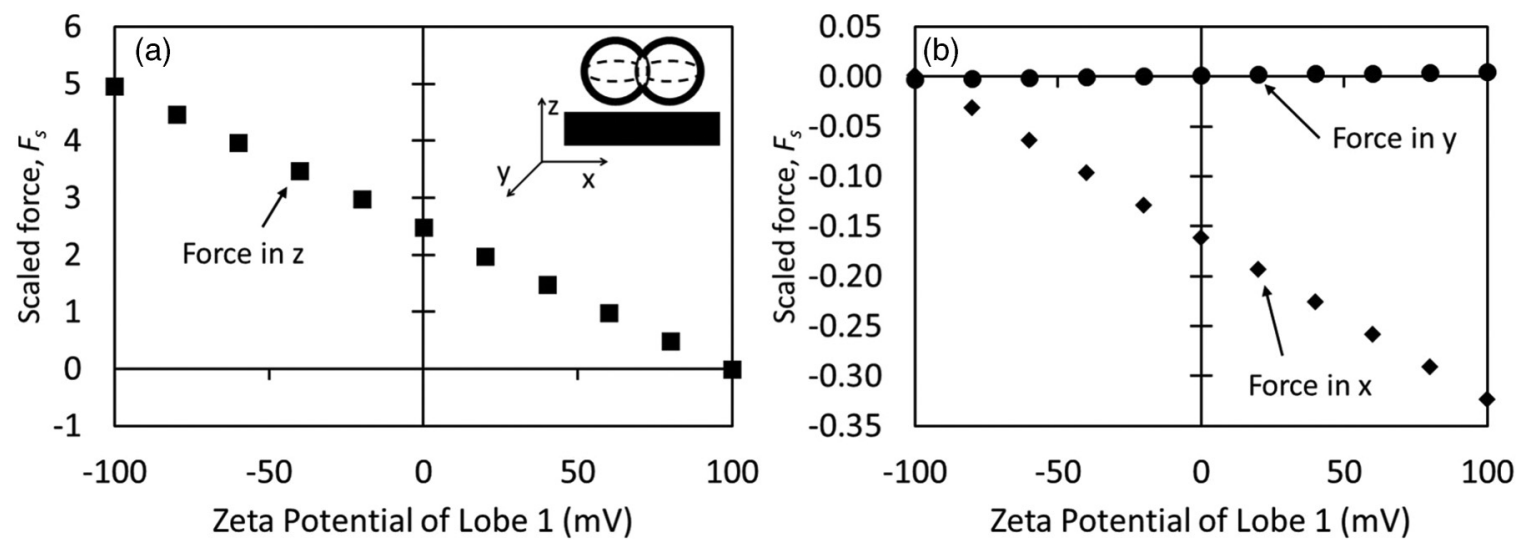

FIG. 5. Force in the (a) $z$ direction and (b) $x$ and $y$ directions on a heterodoublet at a fixed separation distance from a powered electrode. The force on a heterodoublet $\left(R_{1}=R_{2}\right.$ and $\left.\zeta_{1,1} \neq \zeta_{1,2}\right)$ at fixed scaled separation distance $H=0.1$. The $\zeta$ potential of lobe 1 was systematically changed from $-100 \mathrm{mV}$ to $100 \mathrm{mV}$ and the $\zeta$ potential of lobe 2 was fixed at $-100 \mathrm{mV}$. These results show that both forces (in the $z$ and $x$ direction) scale linearly with the difference in $\zeta$ potential at fixed $H$. 

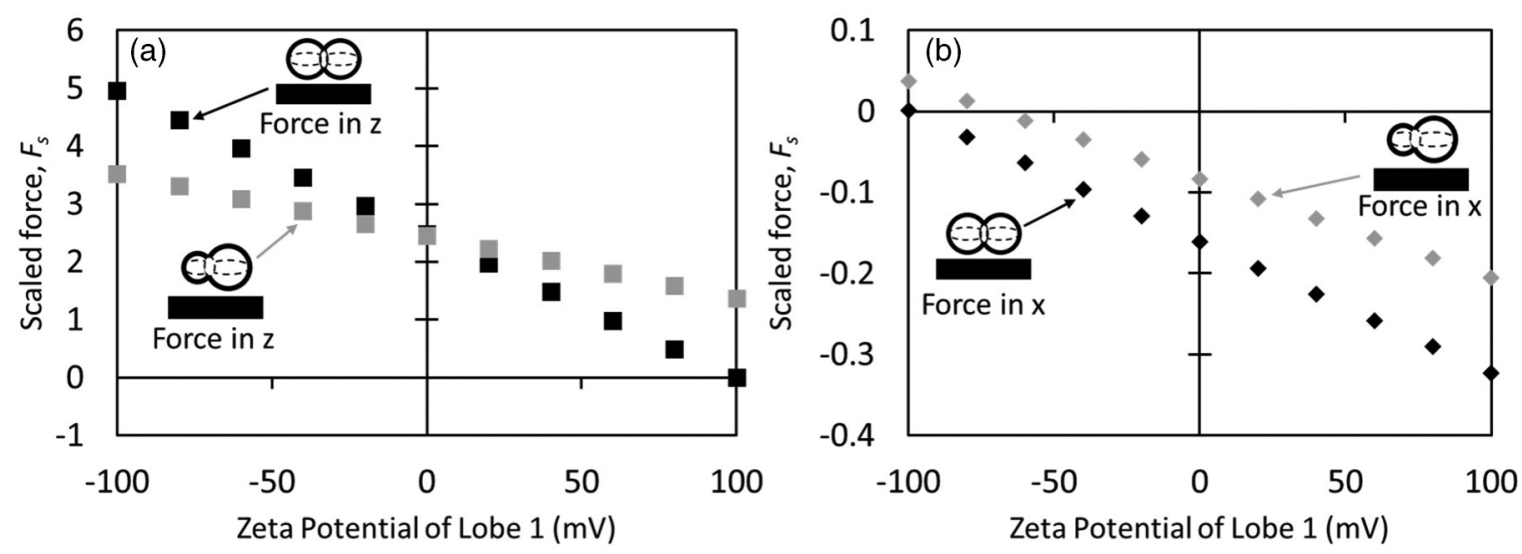

FIG. 6. Force in the (a) $z$ direction and (b) $x$ direction on a heterodoublet at a fixed separation distance from a powered electrode. The force on a heterodoublet $\left(R_{1} \neq R_{2}\right.$ and $\left.\zeta_{1,1} \neq \zeta_{1,2}\right)$ at fixed separation distance $H=0.1$. The $\zeta$ potential of lobe 1 was systematically changed from $-100 \mathrm{mV}$ to $100 \mathrm{mV}$ and the $\zeta$ potential of lobe 2 was fixed at $-100 \mathrm{mV}$ for both $R_{1}=R_{2}$ (black symbols) and $3 R_{1}=2 R_{2}$ (gray symbols). These results show that both forces (in the $z$ and $x$ direction) scale linearly with the difference in $\zeta$ potential at fixed $H$ and a slope that depends on the relative size of the lobes.

that of the equally sized lobe doublet. Further, the vertical force on the heterodoublet with asymmetric lobe size did not go to zero when $\zeta_{1,1}=100 \mathrm{mV}$, which was the condition where the difference in $\zeta$ potential was zero. Although the difference in $\zeta$ potential was $0 \mathrm{mV}$ and the direction of flow was opposite, the magnitude of the electro-osmotic flow fields associated with each lobe were not equal. The downward flow field associated with lobe 1 , which was $\sim 2 / 3$ smaller in radius as compared to lobe 2 , did not fully compensate for the upward flow field associated with lobe 2 . Consequently, there was a finite vertical force for a doublet with equal and opposite $\zeta$ potentials but an asymmetry in lobe size.

Figure 6(b) shows the results for the net force in the $x$ direction from the same calculation. Similar to the results shown in Fig. 5(b), the lateral force in the $x$ direction increased in magnitude as the $\zeta$ potential of lobe 1 increased from $-100 \mathrm{mV}$ to $100 \mathrm{mV}$. However, as was the case for the vertical force, the lateral force on the doublet of asymmetric size scaled with a slightly different slope from that of the doublet with identical lobe size. The net force in the $x$ direction on the doublet of asymmetric size was positive when the lobes were equal to or approximately equal to the same $\zeta$ potential, crossed zero at $\zeta_{1,1}=-70 \mathrm{mV}$, and then grew more negative as the $\zeta$ potential of lobe 1 increased from $-70 \mathrm{mV}$ to $100 \mathrm{mV}$. The point where $\zeta_{1,1}=\zeta_{1,2}$ is of particular note because the break in symmetry is a consequence of only lobe size rather than $\zeta$ potential. Thus, the particle experienced a net lateral force as a consequence of variations in the strength of the flow field associated with each lobe of different size.

\section{Implications for the directed motion of doublets}

The net lateral velocity of doublets was calculated by using an expression previously implemented for the propulsion of doublets neighboring a polarized electrode [37]. The velocity of the doublet is proportional to the net force, with the doublet mobility (i.e., inverse friction factor) as the proportionality constant, $U_{d} \sim F / 6 \pi \mu\left(R_{1}+R_{2}\right)$. Figure 7 shows the lateral velocity prediction for heterodoublets as a function of either lobe- $1 \zeta$ potential [Fig. 7(a)] or size [Fig. 7(b)]. The electric field in the simulation was $70 \mathrm{~V} / \mathrm{m}$, which is a typical magnitude for a dc electric field experiment [12]. The
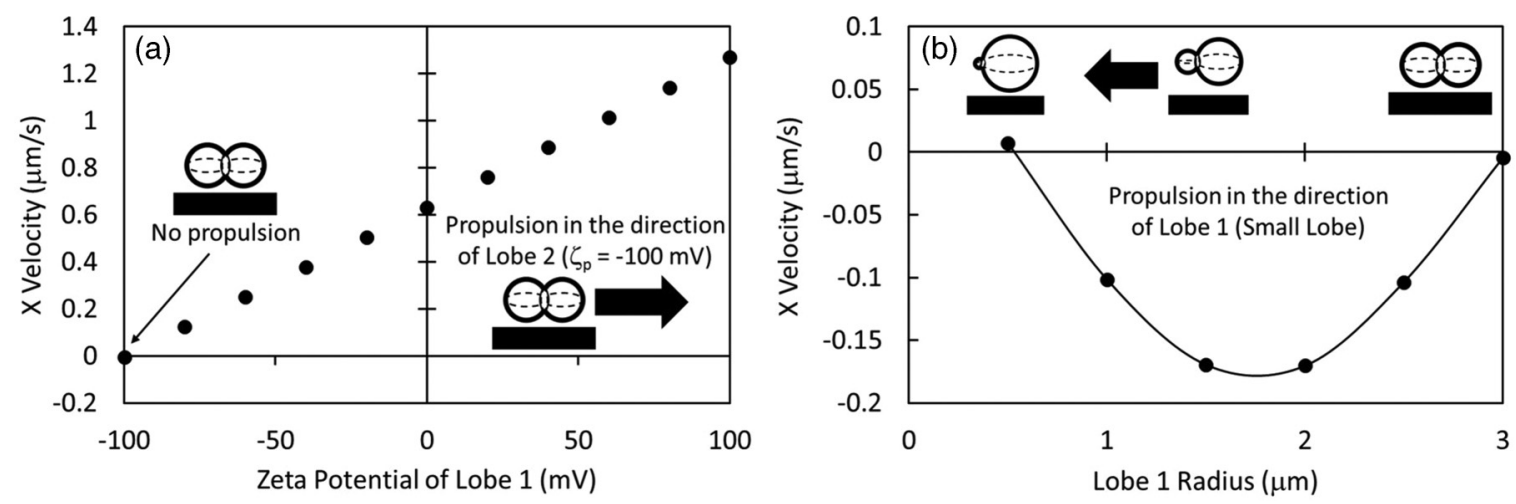

FIG. 7. Velocity predictions as a function of lobe 1 (a) $\zeta$ potential and (b) size. (a) When lobe $1 \zeta$ equaled that of lobe $2(-100 \mathrm{mV})$, the flow field associated with each lobe was upward and along the surface of the particle with no break in symmetry and no apparent velocity. As the $\zeta$ potential of lobe 1 increased, the doublet was propelled in the direction of the lobe with negative $\zeta$ potential. (b) When the radius of lobe 1 equaled that of lobe $2(3 \mu \mathrm{m})$, there was no break in symmetry and no apparent velocity. The doublet was propelled in the direction of the small lobe as the size of lobe 1 decreased. 


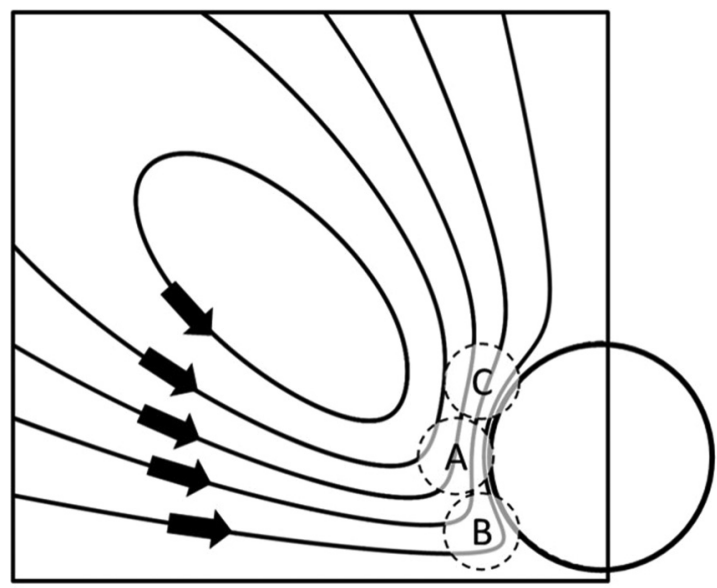

FIG. 8. Streamlines of flow associated with large lobe. The small lobe (lobe 1) is exposed to different flow fields depending on the positions A, B, or C. The lobes have equal center height at position $\mathrm{A}$ and equal separation distance at position B. Position $\mathrm{C}$ was obtained by rotating the doublet clockwise from position $\mathrm{A}$ an amount equal to the angle between positions $\mathrm{B}$ and $\mathrm{A}$.

magnitude of the velocity was approximately $\sim 0.1-1 \mu \mathrm{m} / \mathrm{s}$, which is of the same order of magnitude as that measured during propulsion of colloidal dimers responding to an ac electric field. Note, however, that the mobility of the doublet used to predict the velocity does not include hindrance from the nearby boundary. The boundary will introduce additional hydrodynamic hindrance that will reduce the particle mobility by up to an order of magnitude, thereby reducing the speed by approximately the same amount.

The directionality of doublet motion found in the simulation was consistent with an analysis considering each lobe's response to the attached lobe's flow field (i.e., lobe 1 responding to the flow from lobe 2 and lobe 2 responding to the flow from lobe 1). For example, if lobe 1 is exposed to an "attractive" flow (towards lobe 2) and lobe 2 is exposed to a "repulsive" flow (away from lobe 1), the lobes will move in the direction of lobe 2. A doublet with a positive and negative $\zeta$ lobe will induce repulsive and attractive flow, respectively, thereby inducing net motion towards the lobe with negative $\zeta$ potential. This reasoning works well for a heterodoublet of equal lobe size but unequal $\zeta$ potential [see Fig. 7(a)]. The origin of the directionality for both cases shown in Fig. 7 is consistent with previous work that analyzes a doublet's response by approximating the doublet as a dumbbell [37]. However, this reasoning ignores the difference in flow direction at different positions in a given lobe's streamlines, which becomes important when considering a size asymmetric doublet.

Figure 8 shows the streamlines positioned at equal value of stream function for a single spherical particle. The doublet of asymmetric size is shown in Fig. 8 to have three positions: $\mathrm{A}, \mathrm{B}$, and $\mathrm{C}$. The lobes have equal center height at position $\mathrm{A}$ and equal separation distance at position $\mathrm{B}$. Position $\mathrm{C}$ was obtained by taking a mirror image of position $\mathrm{B}$. The small lobe (lobe 1) was exposed to variations in the large lobe's (lobe 2) flow field through the variations in positions-A, B, and $C$. Lobe 1 does not experience a strong attractive flow when positioned to have equal center height (position A in Fig. 8). However, when the heterodoublet is instead positioned such that each lobe has equal separation distance (position $B$ in Fig. 8), lobe 1 experienced a stronger attractive flow to lobe 2 as $R_{1}$ decreased. At position $\mathrm{C}$, lobe 1 experienced a small net repulsive flow.

Figure 9(a) shows the apparent lateral velocity for a size asymmetric doublet of equal lobe $\zeta$ potential at different orientations. The doublet orientation had a significant effect on the sign and magnitude of velocity. A heterodoublet with unequal lobe size, equal $\zeta$ potential, and equal separation distance (i.e., position B) will propel in the direction of the large lobe, which is consistent with the previously published work [37], considering that both lobes have "attractive" ECEO flow upward and along the lobe surface. However, this same doublet will propel in the direction of the small lobe when oriented at either equal center height (position A) or upward diagonal (position $\mathrm{C}$ ).

Figure 9(b) shows the angular velocity of the doublet as predicted from the net torque at different orientations. The doublet will rotate in the clockwise direction for nearly all orientations and size ratios considered herein, suggesting that the stable orientation for these cases is when the small lobe is positioned above the larger lobe. However, these data suggest that when the small lobe equals or exceeds $2 \mu \mathrm{m}$
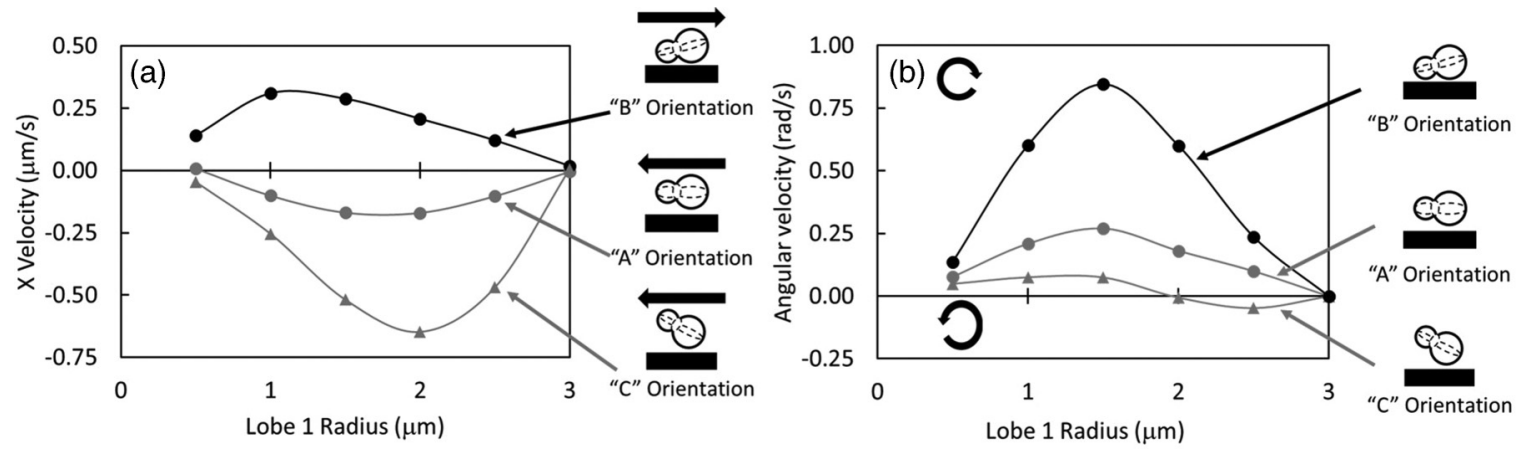

FIG. 9. Dependence of apparent (a) translational and (b) angular velocity on doublet angle. (a) The doublet was propelled in the direction of the small lobe when the centers were at equal height (A) and the doublet was oriented upward (C), but the doublet was propelled in the direction of the big lobe when each lobe had equal separation distance (B). (b) The doublet will rotate in the clockwise direction for all orientations such that the smaller lobe will position above the larger lobe. However, once the doublet reaches a size ratio of 2:3 $\mu \mathrm{m}$, rotation is stabilized or reversed to the counterclockwise direction. 
in radius, there will be a second stable configuration when the doublet is tilted. A doublet will likely sample each of these positions illustrated in Fig. 9 as a consequence of either translational or rotational Brownian motion in an experiment. Position B, with lobes at approximately equal separation distance, will likely be the most common orientation as a consequence of gravity in the absence of an electric field; however, the authors are unaware of any work that has, either experimentally or theoretically, determined the frequency with which each orientation is sampled. These results suggest that the orientation of the doublet is crucial to determine propulsion direction and magnitude at these conditions. As many of the same phenomena are relevant to doublets exposed to ac electric fields, one would expect that orientation of the doublet also plays a role in the lateral propulsion of doublets responding to those conditions.

\section{CONCLUSIONS}

The electric-field-assisted directed assembly of colloidal spheres has been studied for nearly three decades and, in recent years, focus has shifted to the assembly and propulsion of anisotropic particles in response to electric fields. Specifically, work has shown that a colloidal dimer will propel in a direction perpendicular to an applied ac electric field. This paper summarized results from calculations for the response of homo- and heterodoublets to a dc electric field. Both homo- and heterodoublets experienced a net vertical force, similar to that experienced by a colloidal sphere, which depended on both the asymmetry in $\zeta$ potential and lobe size. Homo- and heterodoublets also experienced a net lateral force in the $x$ direction that was perpendicular to the applied electric field. The lateral force depended on the difference in $\zeta$ potential of the two lobes, the lobe size asymmetry, and also the doublet orientation. The net force was predicted to induce a lateral velocity perpendicular to the applied electric field. The magnitude of the predicted velocity was of the same order of magnitude as has been observed for particles responding to ac electric fields. However, the mobility of the doublet was not corrected for hindrance introduced by the nearby boundary, which could reduce the lateral velocity by as much as an order of magnitude. Additionally, the particle experienced rotation from a net torque that depended on the lobe size asymmetry and also the angle of inclination of the doublet. These calculations demonstrate that lobe $\zeta$ potential, lobe size, and orientation of a colloidal doublet can be tuned to achieve propulsion. Future work will include Brownian dynamics simulations of doublets in response to both dc and ac fields in an effort to determine the impact of translational and rotational Brownian motion on doublet propulsion.

\section{ACKNOWLEDGMENTS}

This work was supported by the Cleveland State University Office of Research Startup Fund and also the Faculty Research Development Fund.

This manuscript was written through the contributions of both authors. Both authors have given approval to the final version of the manuscript.
[1] F. Li, D. P. Josephson, and A. Stein, Angew. Chem. Int. Ed. Engl. 50, 360 (2011).

[2] J. D. Forster, J. G. Park, M. Mittal, H. Noh, C. F. Schreck, C. S. O’Hern, H. Cao, E. M. Furst, and E. R. Dufresne, ACS Nano 5, 6695 (2011).

[3] R. M. Rock, P. J. Sides, and D. C. Prieve, J. Colloid Interface Sci. 403, 142 (2013).

[4] P. J. Sides, C. L. Wirth, and D. C. Prieve, Electrochem. SolidState Lett. 13, F10 (2010).

[5] H. Zhou, L. R. White, and R. D. Tilton, J. Colloid Interface Sci. 285, 179 (2005).

[6] W. D. Ristenpart, O. Vincent, S. Lecuyer, and H. A. Stone, Langmuir 26, 9429 (2010).

[7] T. J. Woehl, K. L. Heatley, C. S. Dutcher, N. H. Talken, and W. D. Ristenpart, Langmuir 30, 4887 (2014).

[8] T. J. Woehl, B. J. Chen, K. L. Heatley, N. H. Talken, S. C. Bukosky, C. S. Dutcher, and W. D. Ristenpart, Phys. Rev. X 5, 011023 (2015).

[9] Y. Solomentsev, M. Böhmer, and J. L. Anderson, Langmuir 13, 6058 (1997).

[10] P. J. Sides, C. L. Wirth, and D. C. Prieve, in Electrophoretic Deposition of Nanomaterials, edited by J. H. Dickerson and A. R. Boccaccini (Springer, New York, 2012).

[11] E. Yariv, J. Fluid Mech. 645, 187 (2010).

[12] S. A. Guelcher, Y. Solomentsev, and J. L. Anderson, Powder Technol. 110, 90 (2000).

[13] D. C. Prieve, P. J. Sides, and C. L. Wirth, Curr. Opin. Colloid Interface Sci. 15, 160 (2010).
[14] E. Yariv and O. Schnitzer, Phys. Rev. E 87, 012310 (2013).

[15] J. A. Fagan, P. J. Sides, and D. C. Prieve, Langmuir 22, 9846 (2006).

[16] J. A. Fagan, P. J. Sides, and D. C. Prieve, Langmuir 21, 1784 (2005).

[17] C. L. Wirth, R. M. Rock, P. J. Sides, and D. C. Prieve, Langmuir 27, 9781 (2011).

[18] W. D. Ristenpart, I. A. Aksay, and D. A. Saville, Phys. Rev. E 69, 021405 (2004).

[19] J. A. Fagan, P. J. Sides, and D. C. Prieve, Langmuir 19, 6627 (2003).

[20] W. D. Ristenpart, I. A. Aksay, and D. A. Saville, J. Fluid Mech. 575, 83 (2007).

[21] C. L. Wirth, P. J. Sides, and D. C. Prieve, Phys. Rev. E 87, 032302 (2013).

[22] J. A. Fagan, P. J. Sides, and D. C. Prieve, Langmuir 18, 7810 (2002).

[23] C. S. Dutcher, T. J. Woehl, N. H. Talken, and W. D. Ristenpart, Phys. Rev. Lett. 111, 128302 (2013).

[24] J. D. Hoggard, P. J. Sides, and D. C. Prieve, Langmuir 23, 6983 (2007).

[25] J. A. Fagan, P. J. Sides, and D. C. Prieve, Langmuir 20, 4823 (2004).

[26] J. D. Hoggard, P. J. Sides, and D. C. Prieve, Langmuir 24, 2977 (2008).

[27] M. Böhmer, Langmuir 12, 5747 (1996).

[28] M. Trau, D. A. Saville, and I. A. Aksay, Langmuir 13, 6375 (1997). 
[29] T. Gong, D. T. Wu, and D. W. M. Marr, Langmuir 18, 10064 (2002).

[30] J. Kim, S. A. Guelcher, S. Garoff, and J. L. Anderson, Adv. Colloid Interface Sci. 96, 131 (2002).

[31] Y. Liu, R.-G. Xie, and X.-Y. Liu, Appl. Phys. Lett. 91, 063105 (2007).

[32] Y. Liu, X.-Y. Liu, and J. Narayanan, J. Phys. Chem. C 111, 995 (2007).

[33] F. Ma, D. T. Wu, and N. Wu, J. Am. Chem. Soc. 135, 7839 (2013).

[34] S. Wang, F. Ma, H. Zhao, and N. Wu, ACS Appl. Mater. Interfaces 6, 4560 (2014).

[35] F. Ma, S. Wang, L. Smith, and N. Wu, Adv. Funct. Mater. 22, 4334 (2012).
[36] F. Ma, S. Wang, H. Zhao, D. T. Wu, and N. Wu, Soft Matter 10, 8349 (2014).

[37] F. Ma, S. Wang, D. T. Wu, and N. Wu, Proc. Natl. Acad. Sci. USA 112, 6307 (2015).

[38] J. Berg, An Introduction to Interfaces and Colloids, 1st ed. (World Scientific, Singapore, 2010).

[39] C. L. Wirth, P. J. Sides, and D. C. Prieve, J. Colloid Interface Sci. 357, 1 (2011).

[40] R. M. Rock, P. J. Sides, and D. C. Prieve, J. Colloid Interface Sci. 393, 306 (2013).

[41] A. M. Yake, R. A. Panella, C. E. Snyder, and D. Velegol, Langmuir 22, 9135 (2006).

[42] M. Zabarankin, Proc. R. Soc. A Math. Phys. Eng. Sci. 463, 2329 (2007). 